\title{
De l'étude des migrations aux mobilités transnationales
}

Entretien conduit par Boris Petric le 28 septembre 2010

\section{Catherine Wihtol de Wenden}

\section{(2) OpenEdition}

\section{Journals}

\section{Édition électronique}

URL : http://journals.openedition.org/transcontinentales/199

DOI : 10.4000/transcontinentales. 199

ISBN : 978-2-7351-1557-0

ISSN : 1775-397X

\section{Éditeur}

Editions de la maison des sciences de l'homme

\section{Édition imprimée}

Date de publication : 31 décembre 2010

ISSN : 1950-1684

\section{Référence électronique}

Catherine Wihtol de Wenden, « De l'étude des migrations aux mobilités transnationales », Transcontinentales [En ligne], 8/9 | 2010, document 12, mis en ligne le 31 décembre 2010, consulté le 08 septembre 2020. URL : http://journals.openedition.org/transcontinentales/199 ; DOI : https:// doi.org/10.4000/transcontinentales.199

\section{Ce document a été généré automatiquement le 8 septembre 2020}

Tous droits réservés 


\title{
De l'étude des migrations aux mobilités transnationales
}

\author{
Entretien conduit par Boris Petric le 28 septembre 2010
}

Catherine Wihtol de Wenden

\section{NOTE DE L'ÉDITEUR}

Catherine Wihtol de Wenden - dewenden@ceri-sciences-po.org - est chercheur au Cnrs et enseigne à Sciences Po, Paris. Elle vient de publier l'ouvrage suivant : Catherine Wihtol de Wenden, La question migratoire au XXI siècle : migrants, réfugiés et relations internationales, Paris, Presses de la Fondation nationale des sciences politiques (FNSP), 2010.

B.P. : Vous venez de publier un ouvrage qui associe questions migratoires et relations internationales. Pourriez-vous nous dire en quoi les transformations des processus migratoires affectent les relations internationales?

Nous sortons d'une situation historique où finalement les relations internationales ont structuré la régulation des migrations. Les relations internationales s'organisent à partir des relations interétatiques et les processus migratoires ont longtemps été conditionnés par cette logique. Les grandes migrations s'inscrivaient dans un contexte où les États avaient un rôle déterminant aussi bien dans l'origine des flux d'émigration que dans la gestion de l'immigration. Les migrations politiques ou les migrations économiques étaient structurées par des rapports entre les États.

Aujourd'hui, on peut dire que nous sommes entrés dans une ère inverse où la figure du migrant a beaucoup changé. Tout d'abord, le migrant est devenu un acteur individuel qui bouscule les frontières des États et qui décide de s'inscrire dans une vie marquée par la mobilité. Il ne peut plus être enfermé uniquement dans un seul registre. Avant, on parlait du migrant économique ou du « réfugié politique ». De nos jours, la démarche migratoire est complexe. Souvent, le migrant ne renonce pas définitivement aux liens qu'il entretient avec sa société d'origine. Il migre pour 
diverses raisons et il appréhende sa trajectoire biographique dans la multiplicité des territoires qu'il fréquente. Je donnerai comme exemple la multiplication des binationaux qui essayent d'être à la fois anglais et indien, français et israélien. La double citoyenneté incarne de nouvelles manières d'être et ces processus identitaires tendent à se développer à travers le globe. Aujourd'hui, 92 pays acceptent la double citoyenneté. On voit bien que la transformation des migrations a des implications sur le rôle de l'État et qu'actuellement l'ordre national traditionnel est subverti par ces nouvelles formes de migration. On constate que le monde est complètement pris dans un « réseautage » qui fait que l'État et ses institutions doivent se repositionner par rapports à ces changements. La conséquence majeure est que l'État souverain n'est plus le seul acteur des politiques migratoires.

La question migratoire reste cependant un enjeu international à plusieurs titres. Certains États se sont lancés par exemple dans des politiques volontaristes pour attirer des populations. C'est ce que j'appelle les formes de "désenchevêtrement " des nationalités où des populations se (ré)installent dans un territoire mythifié. Je pense à la politique grecque ayant favorisé le retour des Grecs du Pont-Euxin vivant dans l'Ex-URSS ou, encore, à la politique de l'État allemand facilitant le retour des Aussliedler, c'est-à-dire de tous les Allemands installés en dehors de l'Allemagne notamment ceux que l'on appelle les Allemands de la Volga qui habitaient dans les anciennes Républiques soviétiques. Il y a ainsi des logiques de séduction pour repeupler ou faire face au vieillissement de la population. Il peut même y avoir des logiques de captation électorale et d'influence. Dans cette perspective, on peut interpréter la politique du gouvernement Berlusconi qui s'est lancé dans la délivrance massive de passeports pour les Argentins d'origine transalpine : c'est aussi une manière de vendre l'Italie à l'extérieur et d'accroître son influence dans le monde.

Un autre exemple est celui de la Hongrie, qui lance une politique volontariste en attribuant la citoyenneté aux minorités hongroises vivant dans les pays limitrophes. Cette décision a créé des tensions entre les différents gouvernements de la région. Individuellement, c'est une opportunité pour tous ceux qui ne sont pas encore européens et qui peuvent ainsi circuler beaucoup plus facilement avec un passeport hongrois.

On pourrait également évoquer tout un ensemble de pays qui font de la question migratoire un argument politique pour peser dans les rapports de force qu'ils ont avec d'autres États dans notre monde de plus en plus globalisé. La Libye profite par exemple de sa situation de pays de transit entre l'Afrique et l'Europe. Le colonel Kadhafi utilise ainsi la question migratoire comme un moyen de pression face à la puissance de l'Europe et de certains États.

En fait, en observant la question migratoire on voit bien comment les relations internationales ont profondément été transformées ces dernières décennies.

B.P. : La mobilité devient une manière d'être ayant des implications concrètes sur les rapports sociaux. Pouvez-vous tracer les grandes lignes des changements migratoires?

Nous sommes dans un monde de plus en plus transversal, c'est-à-dire que de nombreux hommes et femmes vivent dans plusieurs sociétés à différents moments de leur vie ou à différentes saisons. La mobilité devient un mode de vie à plusieurs échelles et les gens vivent plusieurs réalités sociales parfois très différentes. 
Beaucoup de ces migrants vivent ici et là-bas pourrait-on dire. On ne peut plus les enfermer dans le modèle du migrant déraciné ayant totalement coupé la relation avec sa société d'origine. Le développement des moyens de communication et des moyens de transport permet d'installer la mobilité comme un mode de vie à part entière. Ces circulations ont des causes multiples, elles peuvent bien sûr être économiques mais elles s'élaborent également dans des motivations identitaires, sociales, religieuses et autres. Ce phénomène touche quasiment toutes les couches sociales.

La grande différence, surtout pour l'Europe, est qu'aujourd'hui la figure du migrant change. La migration est désormais valorisée. Dans l'imaginaire des sociétés de départ circulent de nombreuses histoires de réussite. Et, si pendant longtemps des pays ne se souciaient pas de ces migrants, aujourd'hui de nombreux États leur accordent une attention particulière. Certains soutiennent par exemple des associations diasporiques, en autorisant la double nationalité, en favorisant les procédures administratives pour les papiers d'identité, en facilitant la circulation de l'argent. Le migrant n'est plus la figure honteuse d'une société, il peut au contraire incarner le symbole de la réussite, celui qui est dans le coup, qui prend des risques et qui connaît une forme de mobilité sociale. Des États diasporiques se caractérisent ainsi par la puissance de leurs migrants qui, tout en vivant à l'extérieur, entretiennent une relation étroite avec leur société d'origine. C'est par exemple le cas de l'Érythrée mais aussi des Philippines avec leur cohorte de remittances qui envoient notamment des sommes d'argent considérables au pays.

B.P. : Vous insistez sur le repositionnement de l'État dans son rôle de régulation des flux migratoires. Quels sont, selon vous, les autres acteurs qui jouent un rôle de plus en plus déterminant?

Aujourd'hui, l'État n'est plus le seul acteur, mais nous ne sommes pas pour autant dans un monde uniquement fait d'initiatives individuelles. Il y a, selon moi, deux acteurs déterminants dans les nouveaux processus. Tout d'abord, les associations, les ONG, les communautés peuvent faciliter les départs ou les arrivées des migrants. Ensuite, il y a le rôle des organisations internationales et régionales où de nombreux aspects sont discutés et négociés. Et, il faut dire que ce qui se joue au niveau mondial est bien la question de la gestion de la frontière, de son organisation et de son rôle dans la régulation ou la dérégulation des flux migratoires.

On voit clairement apparaître un phénomène presque contradictoire. D'un côté, des pays durcissent leur politique migratoire comme si l'État voulait affirmer son rôle premier et souverain sur cette question. De nombreux pays consacrent ainsi des budgets très importants à des politiques publiques sécuritaires pour endiguer et limiter des flux migratoires qu'ils ne contrôleraient pas.

De l'autre côté, on voit apparaître la montée en puissance d'une opinion qui considère que l'approche étatique est dépassée. La mobilité devient une valeur indispensable pour multiplier les échanges sociaux à l'échelle planétaire. Dans un rapport du Programme des Nations unies pour le développement (PNUD) de 2009, l'agence de développement de l'ONu met en valeur la mobilité comme facteur indispensable du développement humain. Cet éloge de la mobilité est de plus en plus porté au niveau mondial et devient un nouveau droit humain. De nombreuses organisations transnationales, des ONG, militent pour faire de la mobilité une valeur universelle. Pourtant, comme je l'ai dit, des États restent sur des logiques totalement 
inverses. Ce sont bien sûr les plus puissants, comme les États-Unis, l'Europe, le Canada ou l'Australie, qui mettent en place de nombreuses barrières ou obstacles pour contrôler la mobilité à l'intérieur de leur territoire.

Si nous revenons à une comparaison historique, je dirais que, jusqu'à la moitié du $\mathrm{xx}^{\mathrm{e}}$ siècle, il était difficile de sortir de son pays et il était très facile de rentrer quelque part. On peut prendre l'exemple des multiples expériences sociales des Européens. Ils sont massivement sortis de chez eux pour coloniser, pour évangéliser, pour chercher des ressources économiques ou créer le Nouveau Monde. Il y avait donc de multiples opportunités pour s'installer ailleurs même s'il faut rappeler que les sociétés d'accueil avaient la caractéristique d'être soumises aux logiques impériales de l'époque.

Aujourd'hui, c'est le contraire : pour beaucoup, il est facile de partir de chez soi, mais très difficile d'entrer quelque part.

B.P. : Les politiques publiques des grands États vous paraissent-elles à la hauteur des défis que représente cette accélération des circulations des personnes?

La question migratoire ne peut plus être traitée uniquement par les États et cette révolution date uniquement des années 2000. Les politiques migratoires ont été conçues à une époque qui correspondait à des logiques aujourd'hui dépassées. Il y a eu le temps des migrations économiques ou des migrations liées à des questions politiques donnant un statut singulier aux migrants comme celui, par exemple, de réfugié politique. Aujourd'hui, dans sa biographie, un migrant peut passer par plus de quatre statuts différents. Dans sa trajectoire migratoire, il commence avec le statut de simple touriste et passe à celui de sans-papiers, puis il obtient une carte de séjour temporaire et peut finir par avoir la citoyenneté. Les migrants appellent d'ailleurs cela des carrières administratives.

Les États sont aujourd'hui confrontés au phénomène des sans-papiers qui est bien loin de se limiter à la société française. Les grands pôles migratoires comme la Russie, avec les migrations d'Asie centrale et du Caucase, le Maghreb, avec les migrants de l'Afrique subsaharienne, se trouvent dans des situations d'une grande complexité. Ce sont à la fois des pays de départ, de transit et d'accueil. La grande majorité des politiques migratoires des États n'ont plus rien à voir avec la fluidité des situations actuelles et je dirais qu'aujourd'hui, toutes les régions sont concernées par les logiques circulatoires. Il faut bien voir que le phénomène dans son ampleur est considérable. Les opinions et les décideurs de politiques migratoires, notamment en Europe, ne sont pas conscients de ces changements.

B.P. : Dans les perspectives du $\mathrm{xxl}^{\mathrm{e}}$ siècle, on parle beaucoup des migrations écologiques. Quelle serait la singularité de ce phénomène?

Aujourd'hui, la plupart des statistiques mondiales évoquent le chiffre de 214 millions de migrants à l'échelle internationale dont 51 millions se réalisent dans des échanges Nord/Nord. Il y a par ailleurs les Européens du Nord qui souhaitent vivre au soleil ou les jeunes en quête d'emplois qui partent s'installer à Londres. On voit bien que le processus touche tous les pays et toutes les couches sociales. Cela ne veut pas dire que de nouvelles formes d'inégalités n'apparaissent pas. Nous devons étudier tout autant des formes migratoires qui s'inscrivent dans des relations Nord/Sud que l'inverse. Aujourd'hui, il faut savoir que de très nombreux retraités d'Europe du Nord s'installent en Europe du Sud, au Maroc, au Sénégal ou en Tunisie. Ce phénomène 
existe aussi en Amérique avec des retraités aisés qui vont dans les pays limitrophes pour bénéficier de services et d'une qualité de vie qu'ils ne peuvent pas s'offrir dans leur société. On pourrait également parler des expatriés représentant une forme migratoire très plastique mais qui s'inscrit aussi dans des bouleversements sociaux très importants.

Le développement du tourisme international prend des proportions considérables avec des effets sociaux patents. Finalement, nous devons passer d'une réflexion sur les migrations à une analyse sur les multiples circulations et leurs conséquences sociale, culturelle et politique.

B.P. : La circulation apparaît comme une valeur positive dans un monde globalisé mais cela signifie-t-il pour autant que tout le monde circule?

La circulation est associée à la modernité. Être dans les avions, les transports, est une démonstration de son intégration dans un monde globalisé. Il faut néanmoins se rappeler ceux qui ne peuvent pas bouger, et sont coincés derrière des frontières ou des murs. Cela entraîne la multiplication de nouveaux territoires incertains telles les zones de passage comme Ceuta et Melilla en Europe ou le no man's land entre les ÉtatsUnis et le Mexique. On pourrait évoquer aussi la situation de la frontière forteresse érigée par New Delhi pour se prémunir d'un déferlement de population du Bangladesh en cas d'inondation.

On assiste à la multiplication du phénomène des camps pour "parquer " certaines populations. Il y a les camps de rétention mais aussi les camps de réfugiés. Michel Agier en dénombre plus de 1000 dans le monde. Des gens poussés par des situations politiques ou économiques bougent et sont enfermés parfois pendant des années. Il y aurait aujourd'hui plus de 13 millions d'apatrides dans le monde. Reflet d'une sorte de chaos international où les États ne maîtrisent plus l'ensemble du phénomène.

B.P. : Compte tenu de ces changements, quelles réflexions portez-vous sur l'évolution des études migratoires qui s'appuient sur des catégories statistiques?

L'approche statistique permet d'avoir une vision quantitative du phénomène. Cependant, deux tiers des pays du monde ne fournissent pas de statistiques, c'est-àdire qu'ils ne comptabilisent pas les entrées et les sorties. Nous sommes confrontés aussi aux différentes traditions scientifiques et bureaucratiques nationales. Des pays comme les États anglo-saxons n'opèrent pas de distinction entre nationaux et étrangers, mais prennent plutôt en compte un indicateur permettant de faire la distinction entre le nombre de personnes nées sur leur territoire et celui de personnes nées à l'étranger vivant sur leur territoire. Les pays d'Amérique du Nord, l'Australie ou le Canada choisissent la première option tandis que les pays européens, avec des nuances, optent davantage pour un décompte opposant nationaux et étrangers. L'État demeure alors prépondérant notamment par rapport à sa conception de la citoyenneté.

Les études statistiques doivent être couplées à des études qualitatives. J'ai cité plusieurs exemples concernant la nécessité de connaître les transformations d'ordre culturel, social ou politique des phénomènes migratoires. J'adore, pour ma part, le terrain et je continue à mener des enquêtes qualitatives. J'ai commencé chez Renault, puis dans l'armée, et je poursuis d'autres chemins. Je travaille actuellement sur les migrations chinoises intérieures et je m'intéresse à la multipolarisation des nouvelles figures de la mobilité. 
Il existe aujourd'hui des situations assez étranges mal étudiées. Ainsi, la question de la présence chinoise en Afrique est un bon exemple d'une forme de migration nouvelle. Les chinois débarquent avec leur main-d'œuvre et n'ont presque aucun contact avec la population locale. Les migrations de passage ont existé dans certaines situations historiques notamment dans l'agriculture. Il y a toujours des logiques d'insertion et de mobilité qui nous renseignent aussi sur l'évolution des liens sociaux. On oublie souvent que dans les trajectoires migratoires, beaucoup de migrants décident de repartir. Il faut savoir par exemple qu'aux États-Unis, un tiers des Italiens sont rentrés chez eux.

B.P. : Que pensez-vous des défis que doit relever l'Europe par rapport à ce phénomène qui vient d'être largement mis en débat avec la question de la présence des Roms en France?

Les Roms seraient actuellement plus de 12 millions en Europe. En Roumanie, ils représentent $10 \%$ de la population comme probablement en Bulgarie. Ces Roms sont des ressortissants roumains, c'est-à-dire des citoyens européens qui bénéficient du principe de la libre circulation octroyée depuis peu à la Roumanie. La France profite d'une situation intermédiaire qui fait suite au moratoire limitant la libre circulation des personnes. En 2014, les Roumains auront le droit de s'installer et de travailler en France. L'Europe est dans la situation difficile où la mémoire de la deuxième guerre mondiale pèse énormément car il faut rappeler que les Roms ont été victimes des politiques génocidaires pendant cette période.

Actuellement, en France, il y a beaucoup de confusion sur l'usage des termes : Roms, Roumains et gens du voyage sont mélangés. Il faut savoir que les Roms originaires de Roumanie sont déjà victimes de discrimination dans leur propre pays et la première chose à faire, me semble-t-il, est d'être attentif dans le cadre européen à leur insertion dans leur société. En même temps, la société française a une vision très caricaturale et réductrice de cette communauté que l'on ne peut pas limiter à une population pauvre. Finalement, l'Europe, avec près de 500 millions d'habitants, doit être capable d'accepter et intégrer cette population, sinon c'est inquiétant !

Cela pose un problème plus large qui touche à la question de la citoyenneté. On ne sait pas encore définir la citoyenneté en intégrant la mobilité. Tout cela dépend peutêtre trop de l'ancrage au territoire.

B.P. : On parle beaucoup d'un défi majeur pour le $x x^{e}$ siècle, celui des migrations environnementales. Qu'en pensez vous?

On parle aujourd'hui de déplacés environnementaux comme s'il s'agissait d'un phénomène inédit. C'est important de prendre un peu de distance historique et de rappeler qu'à différentes époques et à travers les cultures, l'anticipation ou le traumatisme suscité par un désastre écologique sont des éléments majeurs de l'histoire sociale. Je pense par exemple au Déluge dans la Bible mais, aussi, aux écrits de Voltaire qui évoquent les conséquences dramatiques du tremblement de terre de Lisbonne, en 1758. On pourrait aussi souligner les traces laissées par la disparition de Pompéi dans la mémoire sociale de la culture européenne. Plus près de nous, dans Les Raisins de la colère de Steinbeck, l'évocation du dustball qui pousse certains habitants à fuir l'Oklahoma. Ces migrations peuvent intervenir à l'échelle d'un pays, par exemple aux États-Unis à la suite du passage du cyclone Katrina à La Nouvelle-Orléans. On peut d'ailleurs observer que ce sont les plus pauvres qui sont restés. 
Aujourd'hui, avec les prévisions scientifiques, nous avons le sentiment de pouvoir anticiper les désastres à venir mais il y a aussi une prise de conscience nouvelle de l'opinion que l'activité humaine peut nuire à la nature. La montée du niveau de la mer, la désertification, la fonte des glaciers sont désormais des phénomènes dont l'évolution dépendrait de l'activité humaine. C'est ainsi que certaines îles, comme Tuvalu en Océanie ou les Maldives, sont menacées de disparition. On parle aussi de celle des îles Halligen en Europe du Nord. Il y a plus grave : le Bangladesh surpeuplé qui est menacé par des inondations majeures venant du Gange. Les régions les plus touchées par les catastrophes environnementales se trouvent au sud alors que le réchauffement climatique est provoqué par les pays du nord. Il est évident que les États ne sont pas en mesure de prendre des dispositions pour gérer ces phénomènes. L'urbanisation du sud se matérialise par le développement de grandes métropoles dont beaucoup situées au bord de la mer pourraient être touchées de plein fouet, dans les décennies à venir, par ces changements.

Cela montre, une fois de plus, que l'organisation du monde ne peut plus se penser à l'échelle de l'État. Nous vivons dans un monde aux interdépendances de plus en plus complexes et la question migratoire n'y échappe pas!

B.P. : Un parcours de recherche s'inscrit souvent dans un parcours personnel. Qu'est-ce qui vous a poussé à consacrer votre vie intellectuelle à l'étude des migrations ?

J'ai été très marquée par mon histoire familiale. Mon grand-père appartenait à une minorité allemande vivant dans les pays Baltes. Ils sont partis à une époque de russification vers l'Amérique latine puis, ensuite, ils ont choisi la France. Les questions de réfugiés, de titre de séjour, de migrant, d'insertion, la question de la double allégeance... tout cela faisait partie de notre univers familial et je me suis donc passionnée pour mieux comprendre les migrations. En 1986, j'ai fait ma thèse de sciences politiques avec Georges Lavaud sur les immigrés et la politique en analysant comment les immigrés deviennent un sujet politique. Ensuite, en entrant au Centre d'études et de recherches internationales (CERI), je me suis concentrée beaucoup plus sur des questions internationales en m'intéressant aux migrations Est-Ouest, à la construction de l'Europe puis, peu à peu, à des aspects mondiaux de la migration.

1 Dans mon dernier ouvrage, j'ai voulu resituer les migrations dans les questions internationales, c'est-à-dire comprendre comment les relations internationales ne sont plus des relations d'États à États et montrer la montée du transnationalisme, des nouveaux lieux du débat politique (avec les forums de l'ONu, etc.). C'est une mise en perspective historique et un dialogue autour des grandes questions théoriques qui concerne le champ des migrations. 